\title{
ON THE STRUCTURE OF THE INTERSECTION OF TWO MIDDLE THIRD CANTOR SETS
}

\author{
Gregory J. Davis and Tian-You Hu
}

\begin{abstract}
Motivated by the study of planar homoclinic bifurcations, in this paper we describe how the intersection of two middle third Cantor sets changes as the sets are translated across each other. The resulting description shows that the intersection is never empty; in fact, the intersection can be either finite or infinite in size. We show that when the intersection is finite then the number of points in the intersection will be either $2^{n}$ or $3 \cdot 2^{n}$. We also explore the Hausdorff dimension of the intersection of two middle third Cantor sets as the sets are translated across one another. We show that the Hausdorff dimension of the intersection can take on any value from 0 to $\ln 2 / \ln 3$; in addition, we show that for each Hausdorff dimension, between 0 and $\ln 2 / \ln 3$, there is a dense set of translation parameters for which the intersections have that particular Hausdorff dimension.
\end{abstract}

\section{Dynamical systems and intersecting Cantor sets}

Our motivation to study the intersection of Cantor sets comes from the discipline of Dynamical Systems. In the late 1800's, Poincaré identified a problem common to many nonlinear dynamical systems - how to describe the changes in a dynamical system when a homoclinic bifurcation takes place. This problem is still the center of much work in the theory of dynamical systems (see the recent monograph [9], for example). As a homoclinic bifurcation takes place, the behavior of a deterministic dynamical system can change from being very robust and predictable (with respect to initial conditions) to being completely chaotic.

Over the past 15 years, work has accelerated in the area of homoclinic bifurcations. Several majour theories have been explored in conjunction with the creation and destruction of homoclinic bifurcations. Along with the possibility of strange attractors, some of the phenomena 
associated with homoclinic bifurcations include omega explosions [8], infinitely many coexisting sinks $[\mathbf{7}],[\mathbf{1 0}],[\mathbf{1}]$ and antimonotonicity [4]. At this time, there is no single theory which integrates and predicts the order of occurrence of these phenomena. However, the development of the theories related to each of these phenomena requires the understanding of how certain stable and unstable manifolds intersect as the homoclinic bifurcation takes place. It is known that the intersections of stable and unstable manifolds have the shape of intersecting Cantor sets; because of this fact, it seems that in order to create a theory unifying omega explosions, infinitely many coexisting sinks and antimonotonicity, it is necessary to understand how Cantor sets intersect in general.

The theories of infinitely many sinks and antimonotonicity rely heavily on knowing when stable and unstable manifolds cannot be separated as they slide across one another, while the theory of omega explosions requires that the stable and unstable manifolds seldom intersect as they slide across one another. Each of these theories hold for parameter values close to a given homoclinic bifurcation in a dissipative planar diffeomorphism.

The criterion used for showing that the stable and unstable manifolds cannot be separated is that the product of the thicknesses of the manifolds is greater than one. Newhouse [6] defines the concept of thickness and show that two Cantor sets which have the product of their thicknesses greater than one cannot be separated. A Cantor set $C$ in the line is represented as the difference of an interval $C_{0}$ and an infinite collection $\left\{U_{j}\right\}$ of disjoint open subintervals (also known as gaps) contained in $C_{0}$. More precisely, $C=\bigcap_{i=0}^{\infty} C_{i}$, where $C_{0}$ is the smallest interval containing $C$, and $C_{i}=C_{0}-\bigcup_{j=0}^{i-1} U_{j}$. Such a sequence of sets $\left\{C_{i}\right\}$ is called a defining sequence of $C$. Let $I_{i j}$ for $j=1,2$ be the two components of $C_{i}$ on either side of the gap $U_{i}$ and let $l(J)$ be the length of an interval $J$. The thickness of a defining sequence is defined by:

$$
\tau\left(\left\{C_{i}\right\}\right)=\inf \left\{l\left(I_{i j}\right) / l\left(U_{i}\right): i \geq 1, \text { where } j=1,2\right\} .
$$

The thickness of a Cantor set is then defined by $\tau(C)=\sup \left\{\tau\left(\left\{C_{i}\right\}\right)\right.$ : $\left\{C_{i}\right\}$ is a defining sequence for $\left.C\right\}$. Using this definition, Newhouse proved the following striking lemma:

Lemma. Let $C^{1}$ and $C^{2}$ be two Cantor sets in $R$ such that $\tau\left(C^{1}\right) \tau\left(C^{2}\right)>1$. If $C^{1}$ is not contained in a gap of $C^{2}$ and $C^{2}$ is not contained in a gap of $C^{1}$, then $C^{1} \cap C^{2} \neq \varnothing$. 
On the other hand, the theory of omega explosions uses the criterion that the sum of the limit capacities of the stable and unstable manifolds is less than one. The limit capacity, $d(C)$, of a Cantor set $C$ will be defined as:

$$
\limsup _{\epsilon \rightarrow 0}(\ln n(C, \epsilon) / \ln (1 / \epsilon)),
$$

where $n(C, \epsilon)$ is the minimum number of open intervals of radius $\epsilon$ needed to cover $C$. The limit capacity of a Cantor set is closely related to its Hausdorff dimension (see $[6]$ ).

Williams [11], Kraft [5] and Hunt, Kan, and Yorke [3] have explored the size of the intersections resulting from two Cantor sets being translated across one another. All of these explorations have assumed that the Cantor sets in question have product of thickness greater than one. Williams produced examples to show that the intersection of two Cantor sets can vary from being one point to containing another Cantor set. Independently, Kraft and Hunt et. al. further developed these ideas and determined all pairs of thicknesses for which the intersection may be a single point and all pairs of thicknesses which must contain another Cantor set. They also consider the problem of how often - as one Cantor set is being translated over another one- does the intersection contain another Cantor set.

In this paper it is our goal to completely describe how the intersection of two middle third Cantor sets change as they are translated across each other. Recall the standard construction for the middle third Cantor set. First we let $C_{0}$ be the closed interval $I$ of length 1 . Although we identify $I$ with $[0,1]$, we note that this construction can be carried out in any closed interval of length 1 (or any closed interval in general).

$$
C_{0}: \overline{0} 1
$$

Now remove from $C_{0}$ the open middle third interval, $(1 / 3,2 / 3)$, to obtain $C_{1}=[0,1 / 3] \cup[2 / 3,1]:$

$$
C_{1}: \overline{1 / 3} \quad \overline{2 / 3} \quad 1
$$

Next remove from each closed interval in $C_{1}$ the open middle third interval to obtain $C_{2}=[0,1 / 9] \cup[2 / 9,3 / 9] \cup[6 / 9,7 / 9] \cup[8 / 9,1]$ :

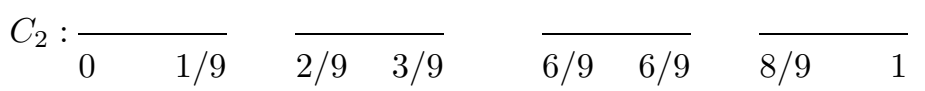

This process is continued indefinitely creating a nested collection of closed nonempty sets $C_{0} \supset C_{1} \supset C_{2} \supset C_{3} \supset \ldots$, where each $C_{n}$ is 
the union of $2^{n}$ intervals, each of length $1 / 3^{n}$. We define the middle third Cantor set to be the infinite intersection:

$$
C=\bigcap_{n=0}^{\infty} C_{n} .
$$

Suppose that two middle third Cantor sets are initially positioned on top of each other as in the following figure:

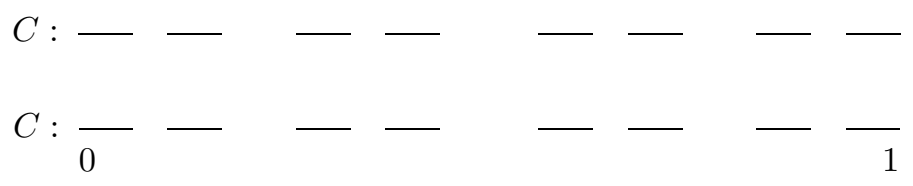

Now translate one Cantor set to the right $x$ units $(x \in[0,1])$ while keeping the other Cantor set in its initial position:

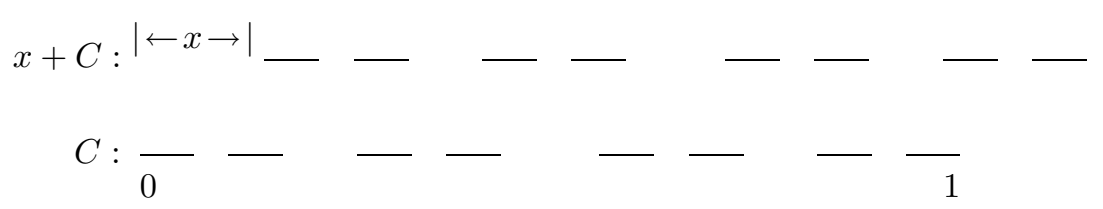

Hence, our goal is to analyze the intersections $(x+C) \cap C$. At first we will consider the problem of how often $x+C$ and $C$ have a nonempty intersection. It can be shown that the thickness of a middle third Cantor set is exactly one, thus Newhouse's lemma (mentioned above) does not apply directly to intersecting middle third Cantor sets. However, by making small modifications to Newhouse's lemma, it can be shown [2] that in the middle third Cantor set context, $(x+C) \cap C \neq \varnothing$ for all $x \in[0,1]$. In this paper we will present an elementary argument, which is independent of Newhouse's, to explicitly show that $(x+C) \cap C \neq \varnothing$ for all $x \in[0,1]$. Our argument, also provides information which allows us to then determine the structure and the size of the intersections of two middle third Cantor sets. In fact, we will completely describe the intersections both in terms of their cardinality and in terms of their Hausdorff dimension. The rest of this paper is organized as follows. Explicit statements of our results are presented in section 2. Section 3 contains proof of the cardinality results and Section 4 contains proofs of the Hausdorff dimension results.

\section{Notation and statement of main results}

The points in the middle third Cantor set $C$ can be conveniently represented by ternary decimals. Recall that for any $x \in[0,1]$ we can express 
$x$ in a ternary decimal expansion:

$$
\begin{aligned}
x=\left(a_{i}\right)= & \sum_{n=0}^{\infty} \frac{a_{n}}{3^{n}}=a_{0} \cdot a_{1} a_{2} a_{3} \ldots \\
& \text { where } a_{0}=0, a_{i}=0,1, \text { or } 2 \text { for all } i>0 .
\end{aligned}
$$

For each nonzero $x$, representation (2.1) is unique except when $x=$ $k / 3^{n}$ and $k$ and $n$ are integers. In these exceptional cases $x$ has two representations:

$$
x=\left(a_{i}\right)=0 \cdot a_{1} a_{2} \ldots a_{n} 000 \ldots\left(a_{n} \neq 0\right)
$$

and

$$
x=\left(a_{i}\right)=0 \cdot a_{1} a_{2} \ldots a_{n-1}\left(a_{n}-1\right) 222 \ldots\left(a_{n} \neq 0\right) .
$$

In order for there to be a $1-1$ correspondence between the $x$ 's in the interval $[0,1]$ and their ternary representations $\left(a_{i}\right)$, we will use representation (2.3) if and only if $a_{n}=1$.

The points in the middle third Cantor set $C$ are characterized by the set of ternary representations which contain no 1's as digits. That is, $x \in C$ if and only if each $a_{i}$ in the representation of $x$ is either a 0 or 2 . If there is an index $n$ such that $a_{n}=1, a_{i}=0$ or 2 for $i<n$ and $a_{i}=0$ (or $a_{i}=2$ ) for all $i>n$, then we will still consider it is a point in $C$, since obviously $a_{n}$ and $a_{i}, i>n$, can be rewritten as either 0 or 2 .

To simplify our notation we will make the following definitions. The segment of digits $a_{r} a_{r+1} \ldots a_{r+k}$, where $a_{r}$ and $a_{r+k}=1$, and for all $i=r+1, \ldots, r+k-1, a_{i}=0$ or 2 is called a $(1,1)$-string of $x$. Furthermore, the $(1,1)$-string sequence of $x$ is defined to be the sequence of all mutually disjoint $(1,1)$-strings of $x$ starting with the first 1 digit of the ternary representation $\left(a_{i}\right)$. If there are an odd number of 1 's in the ternary representation of $x$, then we will make the assumption that there is a 1 at the infinity of the ternary representation to form a final $(1,1)$-string of $x$.

For each $x \in[0,1]$ we will let $T=T(x)$ denote the number of 2's in the $(1,1)$-string sequence of $x$. Similarly we will let $Z=Z(x)$ denote the number of 0 's ( $a_{0}$ does not count) in the complement of the $(1,1)$-string sequence of $x$.

Finally, for each $x \in[0,1]$, we will define the set $C_{x}$ to be:

$$
C_{x}=\{y \in C \mid x+y \in C\} .
$$


Notice that the collection of points in the set $C_{x}$ corresponds exactly to the points in $(x+C) \cap C$. Hence all results stated in terms of $C_{x}$ apply directly to the intersection $(x+C) \cap C$. The phrase $C_{x}$ contains a Cantor set is to mean that $C_{x}=C$ or that $C_{x}$ contains a self similar copy of $C$. Since for $x=0$ and for $x=1$, the structure of $(x+C) \cap C$ is clear, to avoid triviality, we always assume that $x \in(0,1)$ for discussion.

\section{Result A: The cardinality of middle third Cantor set intersec-} tions.

1. Let $x \in(0,1)$ have the expansion $x=0 \cdot a_{1} a_{2} a_{3} \ldots$, then $C_{x}$ contains a Cantor set if and only if either the number of 1's in the expansion is even and all but finitely many of the remaining digits are 0 's, or the number of 1's is odd and all but finitely many of the remaining digits are 2's. All the $x$ 's which satisfy these conditions form a countable, dense subset of $(0,1)$.

2. The number of elements in $C_{x}$, denoted by $\left|C_{x}\right|$, satisfies:

i) $\left|C_{x}\right|=3 \cdot 2^{T+Z-1}$ if and only if the number of 1's in the ternary representation of $x$ is finite and $T+Z<\infty$. All the $x$ 's which satisfy these conditions form a countable, dense subset of $(0,1)$.

ii) $\left|C_{x}\right|=2^{T+Z}$ if and only if the number of 1's in the ternary representation of $x$ is infinite and $T+Z<\infty$. All the $x$ 's which satisfy these conditions form an uncountable, dense subset of $(0,1)$ having Lebesgue measure zero.

iii) $\left|C_{x}\right|$ is uncountably infinite in all other cases. All $x$ 's, with the exception of an uncountable dense subset of $(0,1)$ having Lebesgue measure zero, satisfy these conditions.

\section{Result B: The Hausdorff dimension of middle third Cantor set intersections.}

1. For every $0 \leq \alpha \leq 1$ there exists an $x \in(0,1)$ such that the Hausdorff dimension of $(x+C) \cap C$ satisfies:

$$
\operatorname{dim}[(x+C) \cap C]=(1-\alpha) \frac{\ln 2}{\ln 3} .
$$

2. Let $D_{\alpha}$ be the set of translation parameter $x$ 's where all of the Hausdorff dimensions, $\operatorname{dim}[(x+C) \cap C]$, have the following common value:

$$
D_{\alpha}=\left\{x \in[0,1]: \operatorname{dim}[(x+C) \cap C]=(1-\alpha) \frac{\ln 2}{\ln 3}\right\} .
$$


Then for every $0 \leq \alpha \leq 1$ we have that:

i) $D_{\alpha}$ is a dense subset of $(0,1)$.

ii) Either $m D_{\alpha}=0$ or $m D_{\alpha}=1$, where $m D_{\alpha}$ is the Lebesgue measure of $D_{\alpha}$.

\section{Proof of Result A: The cardinality of the intersection}

Throughout the paper, $x$ will represent an arbitrary number in the interval $(0,1)$. We will identify $x$ with its ternary representation $\left(a_{i}\right)$ where each $a_{i}$ is a 0,1 , or 2 digit for all positive integers $i$. Similarly, $y$ will be any number in the interval $[0,1]$ which is also a point of the middle third Cantor set $C$. We will identify $y$ with its ternary representation $\left(b_{i}\right)$ where each $b_{i}$ is a 0 or 2 digit for each positive integer $i$. We define the indices $I_{k}$ for each positive integer $k$ by:

$$
I_{k}=\max \left\{i \mid 0 \leq i<k, \text { and } a_{i}+b_{i}=0 \text { or } 3\right\}, \quad k=1,2,3, \ldots .
$$

Notice that $I_{k}$ always exists due to the fact that $a_{0}+b_{0}=0+0=0$. The translate of $y$ in $C$ by a distance of $x$ will be given by $t=x+y$. We will identify $t$ with its ternary representation $\left(d_{i}\right)$. Our initial goal is to understand when $t$ is a point of the middle third Cantor set $C$ and when it is not. In the following series of lemmas we will present four conditions which guarantee that a translated of a point from $C$ does not fall on another point of $C$; that is, conditions which imply that $t=x+y$ is not an element of $C$.

Lemma 3.1. If $a_{k}+b_{k}=4$ for some $k \geq 1, a_{i} \neq 1$ for all $I_{k}<i<k$, and $a_{j}+b_{j} \neq 4$ for some $j>I_{k}$, then $t=\left(d_{i}\right) \notin C$.

Proof: By the definition of $I_{k}$ we see that our assumption of $a_{i} \neq 1$ for all $I_{k}<i<k$ implies that $a_{i}+b_{i}=2$ or 4 for all $I_{k}<i<k$. Further, our assumption that $a_{k}+b_{k}=4$ implies that $d_{i}=a_{i}+b_{i}+1(\bmod 3)$ for all $I_{k}<i<k$. Hence, we may conclude that $d_{I_{k}}=1$. We also have that $d_{k}=1$ or 2 since $a_{k}+b_{k}=4$. If we can show that $d_{i}<2$ for some $i>I_{k}$, then $\left(d_{i}\right) \notin C$. The index $j>I_{k}$ for which $a_{j}+b_{j} \neq 4$ can be divided into two cases:

Case 1: $j<k$. As before, we have $a_{j}+b_{j}=2$, hence $d_{j}=0$.

Case 2: $j>k$. Without loss of generality, assume that $j$ is the smallest such index greater than $k$. If $a_{j}+b_{j}=3$, then $d_{j}=0$ or 1 . If $a_{j}+b_{j}<2$, then $d_{j-1}=a_{j-1}+b_{j-1}(\bmod 3)=4(\bmod 3)=1$. Suppose that $a_{j}+$ $b_{j}=2$. Then $d_{j-1}=1$ if $d_{j}=2$. Otherwise, $d_{j}=a_{j}+b_{j}+1(\bmod 3)=0$. In either case we have $t=\left(d_{i}\right) \notin C$. 
Lemma 3.2. If $a_{k}+b_{k}=3$ for some $k \geq 1, a_{i} \neq 1$ for all $I_{k}<i<k$, and $a_{j}+b_{j}>0$ for some $j>k$, then $t=\left(d_{i}\right) \notin C$.

Proof: Repeating the first part of the proof of lemma 3.1 (with $a_{k}+$ $b_{k}=3$ replacing $a_{k}+b_{k}=4$ ), we can again conclude that $d_{I_{k}}=1$. Here the hypothesis that $a_{k}+b_{k}=3$ implies that $d_{k}=0$ or 1 . Without loss of generality, let $j>k$ be the smallest index such that $a_{j}+b_{j}>0$. If $d_{j}>0$, then clearly $\left(d_{i}\right) \notin C$. If $d_{j}=0$, since $a_{j}+b_{j}>0$, so $d_{j-1}=a_{j-1}+b_{j-1}+1(\bmod 3)=0+1=1$. Hence $t=\left(d_{i}\right) \notin C$.

Lemma 3.3. If $a_{j}+b_{j}=a_{k}+b_{k}=1$ for some $j<k, a_{i} \neq 1$ for all $j<i<k$, and $a_{l}+b_{l} \neq 4$ for some $l>k$, then $t=\left(d_{i}\right) \notin C$.

Proof: First we will consider the case in which $a_{i}+b_{i} \leq 2$ for all $j<i<k$. It follows that $d_{j}=1$. Without loss of generality, assume that $l>k$ is the smallest index such that $a_{l}+b_{l} \neq 4$. Notice that $d_{k}=1$ or 2. By considering the cases of $a_{l}+b_{l}$ equals 3 or 2 , or less than 2 , as discussed in the proof of lemma 3.1 , it will imply that $d_{l}=0,1$ or $d_{l-1}=1$. So $t=\left(d_{i}\right) \notin C$.

Now suppose that $a_{i}+b_{i} \geq 3$ for some $j<i<k$. We define $i_{0}$ to be the maximum index between $j$ and $k$ such that $a_{i}+b_{i} \geq 3$. Due to the fact that $a_{i_{0}} \neq 1$, we see that $a_{i_{0}}+b_{i_{0}}=4$ which in turn implies that $d_{i_{0}}=1$. Now, $a_{i}+b_{i} \leq 2$ for all $i_{0}<i<k$. At this point we appeal to the first part of this lemma's proof to conclude that $t=\left(d_{i}\right) \notin C$.

Lemma 3.4. If $a_{j}+b_{j}=1, a_{k}+b_{k}=0$ for some $k>j, a_{i} \neq 1$, for $j<i<k$, and $a_{l}+b_{l}>0$ for some $l>k$ (or if $a_{k-1}+b_{k-1}=2$ ), then $t=\left(d_{i}\right) \notin C$.

Proof: Assume at first that $a_{i}+b_{i} \leq 2$ for all $j<i<k$. This assumption implies that $d_{j}=1$. Note that $d_{k}=0$ or 1 . Thus if $a_{k-1}+$ $b_{k-1}=2$, then $d_{k-1}=2$, hence $\left(d_{i}\right) \notin C$. If $a_{l}+b_{l}>0$ for some $l>k$, without loss of generality, assume that $l$ is the smallest one of such index. If $d_{l}>0$, then $\left(d_{i}\right) \notin C$. If $d_{l}=0$, as in the proof of lemma 3.2, we have $d_{l-1}=1$, so $\left(d_{i}\right) \notin C$.

Now suppose that $a_{i}+b_{i} \geq 3$ for some $j<i<k$. Define $i_{0}$ to be the largest index $i$ between $j$ and $k$ such that $a_{i}+b_{i} \geq 3$, then $d_{i_{0}}=1$ (as in the proof of lemma 3.3). Note that $a_{i}+b_{i} \leq 2$ for all $i_{0}<i<k$, implying $t=\left(d_{i}\right) \notin C$.

Proposition 3.5. Let $x$ be any real number in the interval $(0,1)$ with an even number of 1's in its ternary representation (including no 1's at all in which case $x \in C$ ). If $Z<\infty$, then $\left|C_{x}\right|=3 \cdot 2^{T+Z-1}$. 
Proof: Let $x=\left(a_{i}\right)$ and let $a_{i}=1$ for $i=p_{1}, q_{1}, \ldots, p_{n}, q_{n}$, where $p_{1}<q_{1}<\cdots<p_{n}<q_{n}$. Assume that $a_{i}=0$ or 2 for all $i>q_{n}$ and $i<p_{1}$. By conventions (2.2) and (2.3), there is an $i>q_{n}$ such that $a_{i}=0$, so $Z>0$. Now let $i_{Z}$ be the largest index such that $a_{i_{Z}}=0$. Clearly, $q_{n}<i_{Z}<\infty$ since $Z<\infty$, and $a_{i}=2$ for all $i>i_{Z}$.

We will first show that a necessary condition for $x+y \in C$, where $y=\left(b_{i}\right)$ and each $b_{i}$ is a 0 or 2 is that

$$
b_{p_{i}}=0 \text { and } b_{q_{i}}=2 \text { for } i=1, \ldots, n .
$$

To see that $b_{p_{1}}=0$ we notice that if $b_{p_{1}} \neq 0$, then $a_{p_{1}}+b_{p_{1}}=3$; and then by lemma 3.2 , we would have that $x+y=\left(d_{i}\right) \notin C$. To see that $b_{q_{1}}=2$ we notice that if $b_{q_{1}} \neq 2$, then $b_{q_{1}}=0, a_{p_{1}}+b_{p_{1}}=a_{q_{1}}+b_{q_{1}}=1$ and $a_{i_{Z}}+b_{i_{Z}} \neq 4$; so by lemma 3.3 , we would have that $\left(d_{i}\right) \notin C$. Hence $b_{p_{1}}=0$ and $b_{q_{1}}=2$.

Now suppose that (3.1) is true for $i \leq k-1$. We first show that $b_{p_{k}}=0$. If $b_{p_{k}} \neq 0$, then $a_{p_{k}}+b_{p_{k}}=3$. Since $a_{q_{k-1}}+b_{q_{k-1}}=3$, so $I_{p_{k}} \geq q_{k-1}$ and $a_{i} \neq 1$ for all $I_{p_{k}}<i<p_{k}$. By lemma 3.2, we have $\left(d_{i}\right) \notin C$. So $b_{p_{k}}=0$. Next, we show that $b_{q_{k}}=2$. Suppose not, then $b_{q_{k}}=0$ and $a_{q_{k}}+b_{q_{k}}=1$. Recall that $a_{p_{k}}+b_{p_{k}}=1$ and that $a_{i_{Z}}+b_{i_{Z}}=b_{i_{Z}} \neq 4$, where $i_{Z}>q_{k}$. Applying lemma 3.3 we see that $\left(d_{i}\right) \notin C$. The contradiction shows that $b_{q_{k}}=2$. So (3.1) is true by induction.

Via the following three claims we will examine the remaining choices for the $b_{i}$ 's such that $x+y=\left(d_{i}\right) \in C$ :

Claim 1. Suppose that $i$ is an index such that $0<i<i_{Z}$ and $a_{i}$ is in the complement of the $(1,1)$-string sequece of $x$. If $a_{i}=0$, then $b_{i}$ can be either a 0 or 2 . If $a_{i}=2$, then $b_{i}=0$.

Claim 2. Suppose that $i$ is an index such that $p_{j}<i<q_{j}$, where $j=1, \ldots, n$. If $a_{i}=0$, then $b_{i}=2$. If $a_{i}=2$, then $b_{i}$ can be either a 0 or 2 .

Claim 3. There are exactly three permissible choices for the sequence of $b_{i}$ 's for $i \geq i_{Z}$.

For index $i$ in Claim 1 , we have that $a_{i} \neq 1$ therefore $a_{i}+b_{i}=0,2$ or 4. Since $a_{l}+b_{l}=3$ for $l=q_{j}, j=1, \ldots, n$, we have

$$
I_{i} \geq \begin{cases}0 & \text { if } 0<i<p_{1} \\ q_{j} & \text { if } q_{j}<i<p_{j+1}, \quad j=1, \ldots, n-1 \\ q_{n} & \text { if } q_{n}<i<i_{Z} .\end{cases}
$$


Thus $a_{j} \neq 1$ for all $I_{i}<j<i$. Since $a_{i_{Z}}+b_{i_{Z}} \leq 2$, lemma 3.1 implies that $a_{i}+b_{i} \neq 4$, and thus, $a_{i}+b_{i}=0$ or 2 . Note that $a_{i_{Z}}=0$, so there are $Z-1$ zeros which precede $a_{i_{Z}}$ left in the complement of $x$ 's $(1,1)$-string sequence.

In Claim 2, it follows from lemma 3.4 that for all $i$ such that $p_{j}<i<$ $q_{j}, j=1, \ldots, n$, we must have that $a_{i}+b_{i} \neq 0$ and hence $a_{i}+b_{i}=2$ or 4. Note that $a_{q_{j}}+b_{q_{j}}=3$, thus during the addition of $x$ and $y$ there will be a 1 digit to be carried forward through all indices $i$ where $p_{j}<i \leq q_{j}$ which will make $d_{i}=0$ or 2 . Therefore $b_{i}=2$ if $a_{i}=0$ and $b_{i}$ can be either a 0 or 2 if $a_{i}=2$.

In Claim 3 we first show that either $b_{i}=2$ for all $i>i_{Z}$ or $b_{i}=0$ for all $i>i_{Z}$. Suppose the contrary; without loss of generality we will assume that $b_{r}=0$ and $b_{r+1}=2$ for some $r>i_{Z}$. (The situation where $b_{r}=2$ and $b_{r+1}=0$ for some $r>i_{Z}$ is handled similarly.) We then have that $a_{r+1}+b_{r+1}=4$ and $a_{r}+b_{r}=2 \neq 4$. Notice that $a_{q_{n}}+b_{q_{n}}=3$ thus $q_{n} \leq I_{r+1}<r$. Also notice that $a_{i} \neq 1$ for all $I_{r+1}<i<r+1$. Now lemma 3.1 implies that $\left(d_{i}\right) \notin C$.

To complete the verification of Claim 3 we see that if $b_{i}=2$ for all $i>i_{Z}$, then $b_{i_{Z}}$ must be 0 ; otherwise $a_{i_{z}}+b_{i_{Z}}=2 \neq 4$ and $a_{k}+b_{k}=4$ for any $k>i_{Z}$. Therefore $q_{n} \leq I_{k}<i_{Z}$ and $a_{i} \neq 1$ for all $I_{k}<i<k$. Applying lemma 3.1 will now cause a contradiction. If $b_{i}=0$ for all $i>i_{Z}$, then $d_{i}=2$ for all $i>i_{Z}$ and $d_{i_{Z}}=a_{i_{Z}}+b_{i_{Z}}=0+b_{i_{Z}}=b_{i_{Z}}$; thus, $b_{i_{Z}}$ can be 0 or 2 . It follows that we can have exactly three possibilities for the choices of the sequence of $b_{i}$ 's for $i \geq i_{Z}$.

The results from Claims 1-3 together with the multiplication principle now imply that the total number of choices for the $b_{i}$ such that $t=$ $x+y \in C$ is given by $3 \cdot 2^{T+Z-1}$. If there are no 1 's at all in $x$ 's ternary representation, then $T=0$. Since $0<x<1$, there must be at least one zero in the representation, so $Z>0$. By applying the above argument it is easy to verify that the counting formula for $\left|C_{x}\right|$ will still be valid.

An immediate consequence from the proof of Proposition 3.5 is:

Corollary 3.6. Let $x$ be any real number of the interval $(0,1)$ with an even number of 1 's in its ternary representation. If $Z=Z(x)=\infty$, then $\left|C_{x}\right|$ is uncountably infinite. Furthermore, if all but finitely many digits of the ternary representation of $x$ are 0 's, then $C_{x}$ contains a Cantor set.

Proposition 3.7. Let $x$ be any real number in the interval $(0,1)$. Assume that the number of 1's in the ternary representation of $x$ is odd (in which case the final $(1,1)$-string of $x$ will contain all but finitely many digits). If $T<\infty$, then $\left|C_{x}\right|=3 \cdot 2^{T+Z-1}$. 
Proof: For simplicity we will assume that the number of 1's in the ternary representation of $x$ is 3 ; the general case can be proved similarly as in proposition 3.5. Suppose that $a_{p}=a_{q}=a_{r}=1$, where $p<q<r$. Let $i_{T}$ be the largest index for which $a_{i_{T}}=2$. Clearly $i_{T}$ exists by conventions (2.2) and (2.3); furthermore, $i_{T}>r$, hence $T>0$.

As shown in the proof of proposition 3.5, a necessary condition for $x+y=\left(d_{i}\right) \in C$ is that $b_{p}=0, b_{q}=2$ and $b_{r}=0$.

Via the following three claims we will examine the remaining choices for the $b_{i}$ 's such that $x+y=\left(d_{i}\right) \in C$ :

Claim 1. Suppose that $i$ is an index such that $0<i<p$ or $q<i<r$. If $a_{i}=0$, then $b_{i}$ can be either a 0 or 2 . If $a_{i}=2$, then $b_{i}=0$.

Claim 2. Suppose that $i$ is an index such that $p<i<q$ or $r<i<i_{T}$. If $a_{i}=0$, then $b_{i}=2$. If $a_{i}=2$, then $b_{i}$ can be a 0 or 2 .

Claim 3. There are exactly three permissible choices for the sequence of $b_{i}$ 's for $i \geq i_{T}$.

The verification for Claim 1 is the same as the verification for Claim 1 in the proof of proposition 3.5. Similarly, the verification for the portion of Claim 2 in which $p<i<q$ is the same as the verification for Claim 2 in the proof of proposition 3.5.

To complete the verification of Claim 2 for which $r<i<i_{T}$, we first notice that $a_{r}+b_{r}=1$ and $a_{i}=0$ for all $i>i_{T}$, thus $b_{i}=0$ for all $i>i_{T}$ or $b_{i}=2$ for all $i>i_{T}$, otherwise lemma 3.4 implies that $x+y \notin C$. We will assume, without loss of generality, that $b_{i}=0$ for all $i>i_{T}$. Thus $d_{i}=0$ for all $i>i_{T}$. Also $a_{r}+b_{r}=1$ implies that $a_{i}+b_{i} \neq 0$ for all $r<i<i_{T}$. Otherwise, since $a_{i_{T}}+b_{i_{T}}>0$, lemma 3.4 will imply that $\left(d_{i}\right) \notin C$. So $a_{i}+b_{i}=2$ or 4 for $r<i<i_{T}$. Because $a_{i_{T+1}}+b_{i_{T+1}}=0$, by lemma $3.4 a_{i_{T}}+b_{i_{T}} \neq 2$. Hence $b_{i_{T}}=2$ and $d_{i_{T}}=1$. During the addition of $x$ and $y$ there will be a 1 digit which is carried forward for all $r<i \leq i_{T}$ due to the fact that $a_{i}+b_{i}$ is a 2 or 4 for all $r<i<i_{T}$. Hence we conclude that $d_{i}=0$ or 2 for $r \leq i<i_{T}$.

In Claim 3 we observe that, as above, either $b_{i}=2$ for all $i>i_{T}$ or $b_{i}=0$ for all $i>i_{T}$. If $b_{i}=0$ for all $i>i_{T}$, as argued above, $b_{i_{T}}$ must be 2 . If $b_{i}=2$ for all $i>i_{T}$, then it is easy to see that $b_{i_{T}}$ will be a 2 or 0 . If $b_{i_{T}}=2$, then $d_{i_{T}}=1, d_{i}=0$ or 2 for $r \leq i<i_{T}$ and $d_{i}=2$ for all $i>i_{T}$. If $b_{i_{T}}=0$, then we have that either $d_{r}=1$ and $d_{i}=2$ for all $i>r$, or $d_{r}=2$ and $d_{i_{0}}=1$ for some $r<i_{0}<i_{T}$ and $d_{i}=2$ for all $i>i_{0}$. 
The results from Claims 1-3 together with the multiplication principle imply that $\left|C_{x}\right|=3 \cdot 2^{T+Z-1}$ as desired.

An immediate consequence of the proof of proposition 3.7 is:

Corollary 3.8. Let $x$ be any real number in the interval $(0,1)$. Assume that the number of 1 's in the ternary representation of $x$ is odd. If $T$ is infinite, then $\left|C_{x}\right|$ is uncountably infinite. In addition, if all but finitely many digits of $x$ are 2 's, then $C_{x}$ contains a Cantor set.

Proposition 3.9. Let $x$ be any real number in the interval $(0,1)$. Assume that the number of 1 's in the ternary representation of $x$ is infinite. Also assume that both $Z<\infty$ and $T<\infty$. Then $\left|C_{x}\right|=$ $2^{T+Z}$ and such $x$ form a uncountable dense subset of $(0,1)$ with Lebesgue measure zero.

Proof: Let $a_{i_{j}}=1$ for $j=1,2,3, \ldots$ be the infinitely many 1 's in the ternary representation of $x$. Let $y=\left(b_{i}\right)$ satisfy $x+y \in C_{x}$. As discussed in the proof of proposition 3.5,

$$
b_{i_{j}}= \begin{cases}0 & \text { if } i \text { is odd } \\ 2 & \text { if } j \text { is even }\end{cases}
$$

Since $T$ and $Z$ are finite, there exists an index $k$ such that $a_{i}=0$ for all $a_{i}$ inside of the $(1,1)$-strings of $x$ given by $a_{i_{j}} a_{i_{j}+1} \ldots a_{i_{j+1}}$ for all $j \geq k$. By the same reason as for claim 2 of proposition $3.5, b_{i}$ must be 2 for all such $i$. Furthermore, $a_{i}=2$ for all the $a_{i}$ between the above (1,1)-strings of $x$. Hence $b_{i}=0$ for all such $i$, as in claim 1 of proposition 3.5. So there is exactly one choice for each $b_{i}$ for all $i \geq i_{k}$. Also, we can see that such $\left(a_{i}\right)$ are uncountably many but have Lebesgue measure zero.

We now consider the $b_{i}$ 's where $i<i_{k}$. Here we can reduce our proof to the situation where the number of 1's in the ternary representation of $x$ is even. Using the same argument as we did in the proof of proposition 3.5, we see that the number of choices for the $b_{i}$ 's where $i<i_{k}$ is given by $2^{T+Z}$. Therefore $\left|C_{x}\right|=2^{T+Z}$.

An immediate consequence of the proof of proposition 3.9 is:

Corollary 3.10. Let $x$ be any real number in the interval $(0,1)$. Assume that the number of 1 's in the ternary representation of $x$ is infinite. If either $Z$ or $T$ is infinite, then $\left|C_{x}\right|$ is uncountably infinite.

Proposition 3.11. Let $x$ be any real number in the interval $(0,1)$, then $C_{x}$ contains a Cantor set if and only if either the number of 1's in 
$\left(a_{i}\right)$ is even and all but finitely many of the $a_{i}$ 's are zeros, or the number of 1's in $\left(a_{i}\right)$ is odd and all but finitely many of the $a_{i}$ 's are 2's.

Proof: We only need to prove that the above conditions are necessary as corollaries 3.6 and 3.8 have shown that the conditions are sufficient. Let $x+y=\left(d_{i}\right) \in C_{x}$, then $C_{x}$ contains a Cantor set if and only if each $d_{i}$ can be arbitrarily chosen to be a 0 or 2 starting at some index $i$. We will complete the proof via a contrapositive argument. If $x$ satisfies any one of the following cases, then $C_{x}$ does not contain a Cantor set:

Case 1: The number of 1's is even and $a_{i}=2$ for infinitely many $i$.

Case 2: The number of 1 's is odd and $a_{i}=0$ for infinitely many $i$.

Case 3: The number of 1's is infinite.

In Case 1 , first suppose that $a_{i}=2$ for all $i \geq k$ for some fixed index $k$. If there is a $j>k$ such that $b_{j}=2$ and $b_{j+1}=0$, then $a_{j}+b_{j}=4$ and $a_{j+1}+b_{j+1} \neq 4$. By lemma 3.1, $\left(d_{i}\right) \notin C$. So either $b_{i}=0$ for all $i \geq k_{1}$ or $b_{i}=2$ for all $i \geq k_{1}$ for some index $k_{1}$. Next, if infinitely many 0 's and infinitely many 2 's follow the $(1,1)$-string sequence of $x$, then lemma 3.1 implies that for these $i$ 's, $b_{i}$ must be 0 whenever $a_{i}=2$; therefore, $d_{i}=2$ and will never be 0 . Thus, $C_{x}$ cannot contain a Cantor set for those $x$ in Case 1. Case 2 is handled in the same manner.

In Case 3 , let $a_{i_{j}}=1$ for $j=1,2,3, \ldots$ be the infinitely many 1 's in $\left(a_{i}\right)$. As in the proof of proposition 3.9 we have that

$$
b_{i_{j}}= \begin{cases}0 & \text { if } j \text { is odd } \\ 2 & \text { if } j \text { is even }\end{cases}
$$

It follows that $d_{i_{j}}$ must be 0 if $j$ is even, and therefore, $t=\left(d_{i}\right) \notin C$.

Finally, by combining the results of proposition 3.5 through proposition 3.11 we now have a proof of Result A on the cardinality of middle third Cantor set intersections.

4. Proof of Result B: The Hausdorff dimension of the intersection

Let $n_{1}<n_{2}<\cdots<n_{i}<\cdots$ be any subsequence of the set of positive integers. For each positive integer $k$, we define the index $k^{\prime}$ to be $k^{\prime}=\max \left\{i \mid n_{i} \leq k\right\}$, and set:

$$
\alpha=\alpha\left(n_{1}, n_{2}, \ldots\right)=\limsup _{k \rightarrow \infty} \frac{k^{\prime}}{k} .
$$

Notice that each $\alpha$ satisfies $0 \leq \alpha \leq 1$. For each sequence $n_{1}, n_{2}, \ldots$ and the corresponding $\alpha$, a subset $C^{\alpha}$ of the middle third Cantor set $C$ will 
be formed as follows. $C^{\alpha}$ is made up of all the $x$ 's from $C$ which have digits $a_{i}$ preasigned if the index $i$ is from the sequence $n_{1}, n_{2}, \ldots$; the remaining digits in the ternary representations are arbitrary:

$$
\begin{aligned}
C^{\alpha} & =C^{\alpha}\left(a_{n_{1}}, a_{n_{2}}, \ldots\right) \\
& =\left\{x \in C \mid a_{n_{i}} \text { is preassigned a } 0 \text { or } 2 \text { for all } i\right\} .
\end{aligned}
$$

Let $\operatorname{dim} C^{\alpha}$ denote the Hausdorff dimension of $C^{\alpha}$.

Proposition 4.1. $\operatorname{dim} C^{\alpha}=(1-\alpha) \frac{\ln 2}{\ln 3}$.

Proof: Without loss of generality we can assume that $a_{n_{i}}=0$ for all $i \geq 1$. $C^{\alpha}$ can be constructed by first setting $E_{0}$ to be the interval $[0,1]$. Next we define $E_{1}$ to be the set given by:

$$
E_{1}= \begin{cases}{[0,1 / 3]} & \text { if } n_{1}=1 \\ {[0,1 / 3] \cup[2 / 3,1]} & \text { otherwise. }\end{cases}
$$

Sets $E_{n}$ for $n>1$ will be defined inductively. Suppose that $E_{k}$ is the union of $2^{k-k^{\prime}}$ basic ternary intervals of length $(1 / 3)^{k} \cdot E_{k+1}$ is defined by:

$$
E_{k+1}=\left\{\begin{array}{l}
\bigcup_{[a, b] \subset E_{k}}\left[a, a+(1 / 3)^{k+1}\right] \text { if } n_{i}=k+1 \text { for some } i \\
\bigcup_{[a, b] \subset E_{k}}\left[a, a+(1 / 3)^{k+1}\right] \cup\left[b-(1 / 3)^{k+1}, b\right] \text { otherwise. }
\end{array}\right.
$$

Finally we have that $C^{\alpha}=\bigcap_{n=0}^{\infty} E_{n}$.

We will define a probability measure $P$ on $C^{\alpha}$ by:

$$
P\left(C_{\alpha} \cap I_{k}\right)=(1 / 2)^{k-k^{\prime}}, \quad k=1,2, \ldots
$$

where $I_{k}$ is any basic ternary interval of $E_{k}$.

It is straightforward to verify that $P$ is a well-defined measure. In addition, for each real number $s>0$, we have that:

$$
\frac{P\left(C_{\alpha} \cap I_{k}\right)}{\left(\operatorname{diam} I_{k}\right)^{S}}=\frac{\left(\frac{1}{2}\right)^{k-k^{\prime}}}{\left(\frac{1}{3}\right)^{k s}}=\frac{3^{\left(k^{\prime}-k\right) \frac{\ln 2}{\ln 3}}}{3^{-k s}}=3^{k\left(s-\left(1-\frac{k^{\prime}}{k}\right) \frac{\ln 2}{\ln 3}\right)} .
$$

Next we define $\theta$ to be the nonnegative value given by:

$$
\theta=\liminf _{k \rightarrow \infty}\left(1-\frac{k^{\prime}}{k}\right) \frac{\ln 2}{\ln 3}=(1-\alpha) \frac{\ln 2}{\ln 3},
$$


and observe that:

$$
\limsup _{k \rightarrow \infty} \frac{P\left(C_{\alpha} \cap I_{k}\right)}{\left(\operatorname{diam} I_{k}\right)^{S}}= \begin{cases}0 & \text { if } s<\theta \\ \infty & \text { if } s>\theta .\end{cases}
$$

It now follows that $\operatorname{dim} C^{\alpha}=(1-\alpha) \frac{\ln 2}{\ln 3}$.

Using proposition 4.1, we will study the Hausdorff dimension of $(x+$ $C) \cap C$ for any given $x \in[0,1]$. Let $x$ have the ternary representation $\left(a_{i}\right)$ and let $a_{n_{1}} a_{n_{2}} \ldots a_{n_{i}} \ldots$ be the subsequence of $\left(a_{i}\right)$ consisting of all the 0 's inside the $(1,1)$-string sequence of $x$ and all the 2 's outside the string sequence together with all the 1's in the ternary representation of $x$. It follows from the proofs of propositions 3.5, 3.7 and 3.9 that $(x+C) \cap C$ consists of those $\left(d_{i}\right) \in C$ such that each $d_{n_{i}}$, where $i=1,2, \ldots$, must be a fixed digit, while the rest of the digits in the representation $\left(d_{i}\right)$ can be randomly chosen to be a 0 or 2 . By proposition 4.1 , we now have that:

$$
\operatorname{dim}[(x+C) \cap C]=(1-\alpha) \frac{\ln 2}{\ln 3},
$$

where $\alpha$ is defined by equation (4.1). From the discussion we see that adding a finite number of $(1,1)$-strings to (or deleting from) the beginning of $\left(a_{i}\right)$ is equivalent to adding (or deleting) a finite number of terms to the beginning of the subsequence an $a_{n_{1}} a_{n_{2}} \ldots a_{n_{i}} \ldots$. As a result, the upper limit in (4.1) will be unchanged. We hence have the following corollary.

Corollary 4.2. Let $x=\left(a_{i}\right)$ and $y=\left(b_{i}\right)$ be any two points in $(0,1)$. If there exist integers $m$ and $n$ such that $a_{i}=b_{i+n}$ for all $i>m$ and both $a_{1} \ldots a_{m}$ and $b_{1} \ldots b_{m+n}$ have an even (or odd, respectively) number of 1 's, then

$$
\operatorname{dim}[(y+C) \cap C]=\operatorname{dim}[(x+C) \cap C] .
$$

To continue, we define the set $D_{\alpha}$ to be the set of translation parameters $x$ which correspond to intersections $(x+C) \cap C$ having a common value; more precisely,

$$
D_{\alpha}=\left\{x \in[0,1] \mid \operatorname{dim}[(x+C) \cap C]=(1-\alpha) \frac{\ln 2}{\ln 3}\right\} .
$$

Proposition 4.3. For every $0 \leq \alpha \leq 1, D_{\alpha}$ is a dense subset of $(0,1)$.

Proof: We will first show that $D_{\alpha} \neq \varnothing$. Let $0 \cdot \epsilon_{1} \epsilon_{2}, \ldots, \epsilon_{i} \in\{0, \ldots, 9\}$, be the base 10 decimal expansion of $\alpha$. Clearly $\lim _{n \rightarrow \infty} \frac{\epsilon_{1} \ldots \epsilon_{n}}{10^{n}}=\alpha$. Let 
$x \in C$ be chosen so that in the first $10^{n}$ digits of its ternary representation there are exactly $\epsilon_{1} \ldots \epsilon_{n}$ digits which are 2's and the rest of the digits are 0 's. From the proof of proposition 3.5 we know that the set $(x+C) \cap C$ consists precisely of those $\left(b_{i}\right) \in C$ such that $b_{i}=0$ if $a_{i}=2$ and $b_{i}=0$ or 2 otherwise. It now follows from proposition 4.1 and the construction of $x$ that $x \in D_{\alpha}$ and therefore $D_{\alpha} \neq \varnothing$. Furthermore, if $y$ is obtained by adding a finite number of $(1,1)$-strings to the tail digits of $x$, then corollary 4.2 implies that $y \in D_{\alpha}$. Clearly these $y$ 's form a dense subset of $(0,1)$.

Proposition 4.4. For every $0 \leq \alpha \leq 1$, either $m D_{\alpha}=0$ or $m D_{\alpha}=$ 1. Here $m D_{\alpha}$ is the Lebesgue measure of $D_{\alpha}$.

Proof: Let $\beta \in(0,1)$; we will show that if $m D_{\alpha}>0$ then $m D_{\alpha}>\beta$ and therefore $m D_{\alpha}=1$. Notice that if $m D_{\alpha}>0$, then there exists a ternary interval $I$ of length $3^{-n}$ such that:

$$
1 \geq \frac{m\left(D_{\alpha} \cap I\right)}{m(I)}>\beta .
$$

Proposition 4.4 will be proven if we can show that the interval $I$ in expression (4.2) can be replaced by any ternary interval of length $3^{-n}$ from $[0,1]$. Let $J$ be an arbitrarily chosen ternary interval of length $3^{-n}$ from $[0,1]$. Recall that all points in $I$ (or $J$, respectively) have the same beginning $n$ digits in their ternary expansion.

For any given pair of points $\left(a_{i}\right),\left(b_{i}\right)$ with $\left(a_{i}\right) \in I$ and $\left(b_{i}\right) \in J$, either $\left(a_{i}\right)$ and $\left(b_{i}\right)$ will both have an even (odd) number of 1's in their first $n$ digits or one will have an even number of 1's and the other will have an odd number of 1's in their first $n$ digits.

Suppose that both $a_{1} \ldots a_{n}$ and $b_{1} \ldots b_{n}$ have an even (or odd, respectively) number of 1's. Let $A$ denote the translation of $I \cap D_{\alpha}$ to $J$; that is,

$A=\left\{y_{x} \in J \mid y_{x}=0 \cdot b_{1} \ldots b_{n} a_{n+1} a_{n+2} \ldots\right.$, for all $\left.x=\left(a_{i}\right) \in\left(I \cap D_{\alpha}\right)\right\}$.

The points $x$ and $y_{x}$ satisfy the hypotheses of corollary 4.2 , thus $\operatorname{dim}[(y+$ $C) \cap C]=\alpha$ for all $y \in A$. Therefore $A \subseteq\left(J \cap D_{\alpha}\right)$ and we now see that $m\left(I \cap D_{\alpha}\right)=m(A) \leq m\left(J \cap D_{\alpha}\right)$. Hence expression (4.2) remains true when $I$ is replaced by $J$.

Now suppose that one of the sequences $a_{1} \ldots a_{n}$ or $b_{1} \ldots b_{n}$ has an odd number of 1's and the other sequence has an even number of 1 's. Let $B$ be the union of the similitudes of $I \cap D_{\alpha}$ on every middle third ternary 
interval of $J \backslash C$; that is,

$B=\left\{y_{x} \in J \mid y_{x}=0 \cdot b_{1} \ldots b_{n+k} a_{n+1} a_{n+2}, \ldots\right.$, for all $x=\left(a_{i}\right) \in\left(I \cap D_{\alpha}\right)$, where $b_{n+k}=1, b_{i}=0$ or 2 for $\left.i=n+1, \ldots, n+k-1, k=1,2, \ldots\right\}$.

It is straightforward to verify that $m B=m\left(I \cap D_{\alpha}\right)$. Now by corollary 4.2 we have $\operatorname{dim}[(y+C) \cap C]=\alpha$ for all $y \in B$. So $B \subseteq\left(J \cap D_{\alpha}\right)$ and expression (4.2) remains true when $I$ is replaced by $J$.

By combining the results in this section we obtain a proof of Result B on the Hausdorff dimension of middle third Cantor set intersections.

Acknowledgements. The authors would like to thank the editor and an anonymous reviewer for their careful reading and constructive remarks, which have made this manuscript a stronger contribution.

\section{References}

1. Davis G. J., Infinitely many coexisting sinks from degenerate homoclinic tangencies, A.M.S. Trans. 323(2) (1991), 727-748.

2. Davis G. J., Intersections of middle $\alpha$ Cantor sets, preprint, 1993.

3. Hunt B. R., Kan K. And Yorke J., When Cantor sets intersect thickly, A.M.S. Trans. 339(2) (1993), 869-888.

4. Kan I. AND Yorke J., Antimonotonicity: Concurrent creation and annihilation of periodic orbits, A.M.S. Bull. 23(2) (1990), 469-476.

5. Kraft R. L., Intersection of thick Cantor sets, Memoirs of the A.M.S. 468 (1992).

6. Newhouse S. E., Nondensity of Axiom A(a) on $S^{2}$, Proc. Sympos. Pure Math. 14 (1970), 191-202.

7. Newhouse S. E., The abundance of wild hyperbolic sets and nonsmooth stable sets for diffeomorphisms, IHES Publ. Math. 50 (1979), 101-151.

8. Palis J. And Takens F., Hyperbolicity and the creation of homoclinic orbits, Annals of Math. 125 (1987), 337-374.

9. Palis J. and Takens F., "Hyperbolicity and Sensitive Chaotic Dynamics at Homoclinic Bifurcations," Cambridge Studies in Advanced Mathematics, 1993.

10. Robinson C., Bifurcation to Infinitely many sinks, Comm. Math. Phys. 90 (1983), 433-459.

11. Williams R. F., How big is the intersection of two thick Cantor sets, in "Contemporary Mathematics," M. Brown, ed., Proc. of the 
1989 Joint Summer Research Conference on Continua and Dynamical Systems, A.M.S., Providence R. I., 1991.

\author{
Department of Mathematics \\ University of Wisconsin - Green Bay \\ Green Bay, WI 54311-7001 \\ U.S.A.
}

Primera versió rebuda el 25 d'Agost de 1993, darrera versió rebuda el 7 de Setembre de 1994 